For Internal Distribution Only

Experimental Support and Facilities Division

Collider-Accelerator Department

BROOKHAVEN NATIONAL LABORATORY

Upton, New York 11973

ES\&F Division Technical Note \# 163

\title{
AGS Polarimetry DAQ upgrade and its usage
}

Kin Yip

January 23, 2006 


\title{
AGS Polarimetry DAQ upgrade and its usage
}

\author{
Jan. 18, 2005
}

Kin Yip

Experimental Support and Facilities Division

Collider-Accelerator Department 
The AGS polarimetry data acquisition (DAQ) has been upgraded recently. This upgrade mainly involves replacing the PCI interface with the USB 2.0 protocol. The new USB interface makes use of the FASTCAMAC ${ }^{\mathrm{i}}$ protocol which is an extension of the CAMAC standard. The original PCI interface has used the CC32 controller $^{\text {ii }}$ in the CAMAC chassis connected by a 50-pin cable to a PCIADA card plugged in a PCI slot of the PC cnipol.ags.bnl.gov. After the upgrade, we have used CMC controllers ${ }^{i i i}$ which are connected by USB cables to the USB 2.0 slots on our new PC cnipol2.ags.bnl.gov.

After the upgrade, the new PC is connected to 3 CAMAC chasses. A LeCroy module $2367^{\text {iv }}$ sits in one chassis which is connected to the new PC with the PCI interface. This module mainly controls and synchronizes the timing for the entire DAQ system and it also provides some necessary interrupt functions which the USB protocol cannot yet provide. All the WFD's (WaveForm Digitizers) sit in the other 2 CAMAC chasses which are connected to the same PC with the USB 2.0 cables. Each chassis has 12 WFD's which receive inputs from 48 channels connected to the silicon detectors in the AGS tunnel through pre-amplifier and pulse shaper boards. The WFD's mainly digitize the data they receive and analyze with its algorithm in Xilinc FPGA's to give out the time-of-flight (TOF) and kinetic energy information for the particles hitting the silicon detectors in the AGS tunnel.

Other important changes and features include:

- The operating system in the PC now is now running with the Linux kernel 2.6.9-xxx in the flavor of the free Scientific Linux $(4 . x)^{\mathrm{v}}$.

- The 2 sets of 48 channels (ie. a total of 96 channels) in the 2 CAMAC chasses are read simultaneously by using the method of multiply threaded execution. It has been written in C using POSIX pthread library in Linux.

- The DAQ now sends results (count rates, polarizations etc.) and other information through $\mathrm{CDEV}^{\mathrm{vi}}$ to the PAT page which is written by S. Nemesure in the Control Division. The communication between the DAQ system and the PAT page allows users to do target scan and take polarization measurements.

A couple exercises that have been done using the new DAQ system together with the new shaper boards are described here.

\section{Finding attenuations as a tool to detect faulty lemo $^{\text {vii }}$ connection}

The original purpose was to measure the real attenuations for each channel on the shaper board. This was done by injecting a known pulse voltage from a digital pulse generator (where one can dial in a particular value) and the frequency used was typically 
10K Hz. The output signals were measured by the WFD's (used as ADC's here). The same set of measurements with and without the attenuator (on the shaper board) would give us the attenuation ratios. This is needed because we need to use attenuator for alpha source calibration and the output voltages induced from the alpha source are much stronger those from the proton beam.

One plot of the attenuations ( $\sim 3$ and $\sim 5)$ is shown in Figure 1. One can that there is an odd channel as its attenuation is very different from the others. This has been fixed by replacing the faulty lemo cable which was connected to that particular aforementioned odd channel.

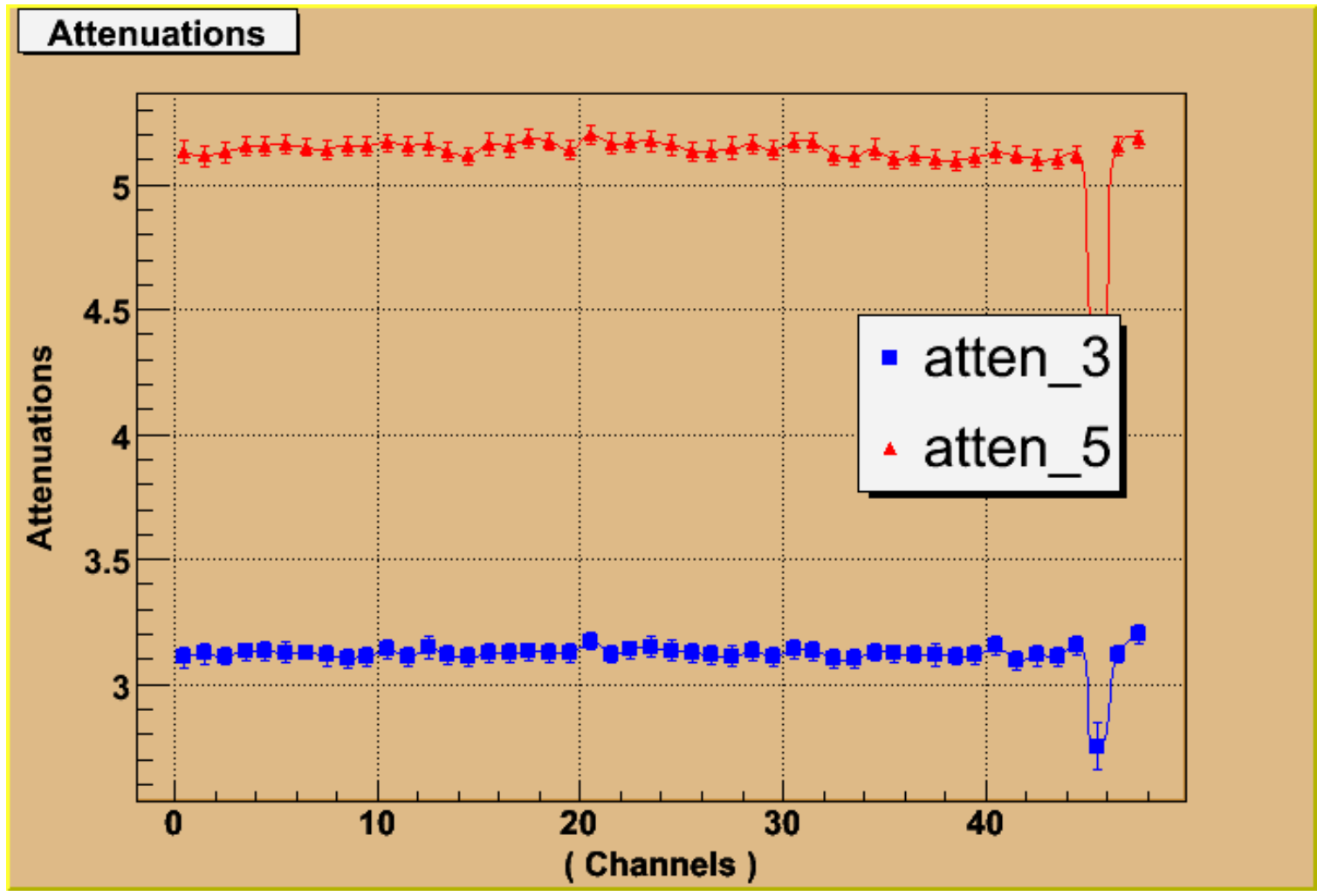

Figure 1: Attenuations measured for the 48 channels of the shaper board using WFD's.

\section{Linearity measurements}

A digital pulse generator has been used in which one can dial a value exactly. This is possible because the shaper boards can accept pulse inputs. Various amplitudes of pulses are injected into the shaper board. A simple schematic diagram is shown in Figure 2.

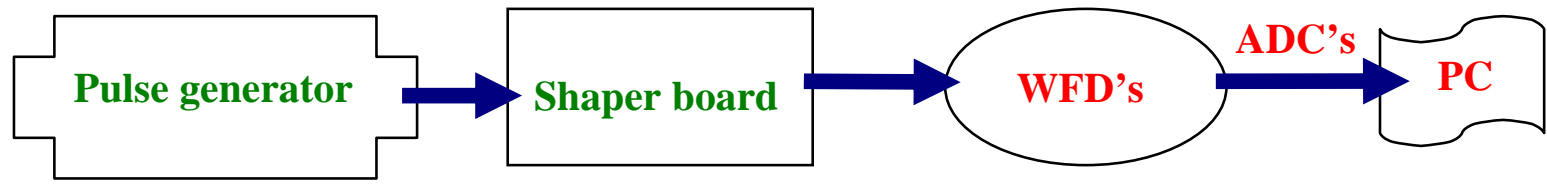


Figure 2: Schematic diagram for the data transfer in the calibration.

We have chosen 8 different input pulse amplitudes at the pulse generator and then collect the ADC values (given by the WFD's) using the DAQ system. The ADC's are plotted against the input pulse amplitudes. The plot for one of the channels is shown in Figure 3. Qualitatively, one can see that the relationship between ADC counts and the input pulse amplitudes is linear.

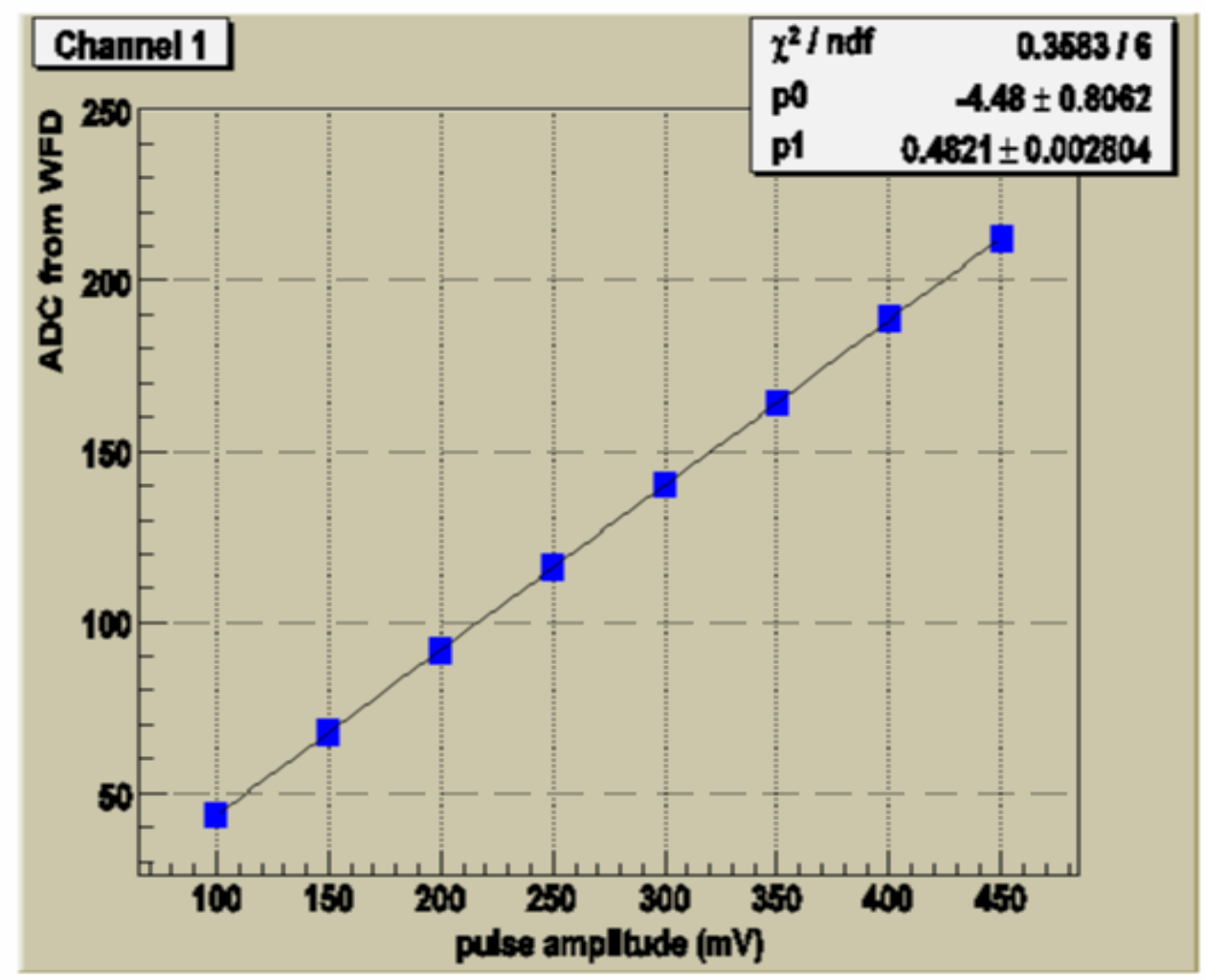

Figure 3: ADC's from the WFD versus input voltages from the pulse generator.

To examine how good the linearity is in 16 channels that I have tested, I have made a histogram of (ADC - ADC_fit)/ADC_fit, where ADC_fit is the fitted value of ADC on the fitted straight line at each input voltage as shown in Figure 3. The result is shown in Figure 4. The standard deviation of the difference plot of ADC ADC_fit)/ADC_fit is about $0.2 \%$ which shows that the linearity is very good. 


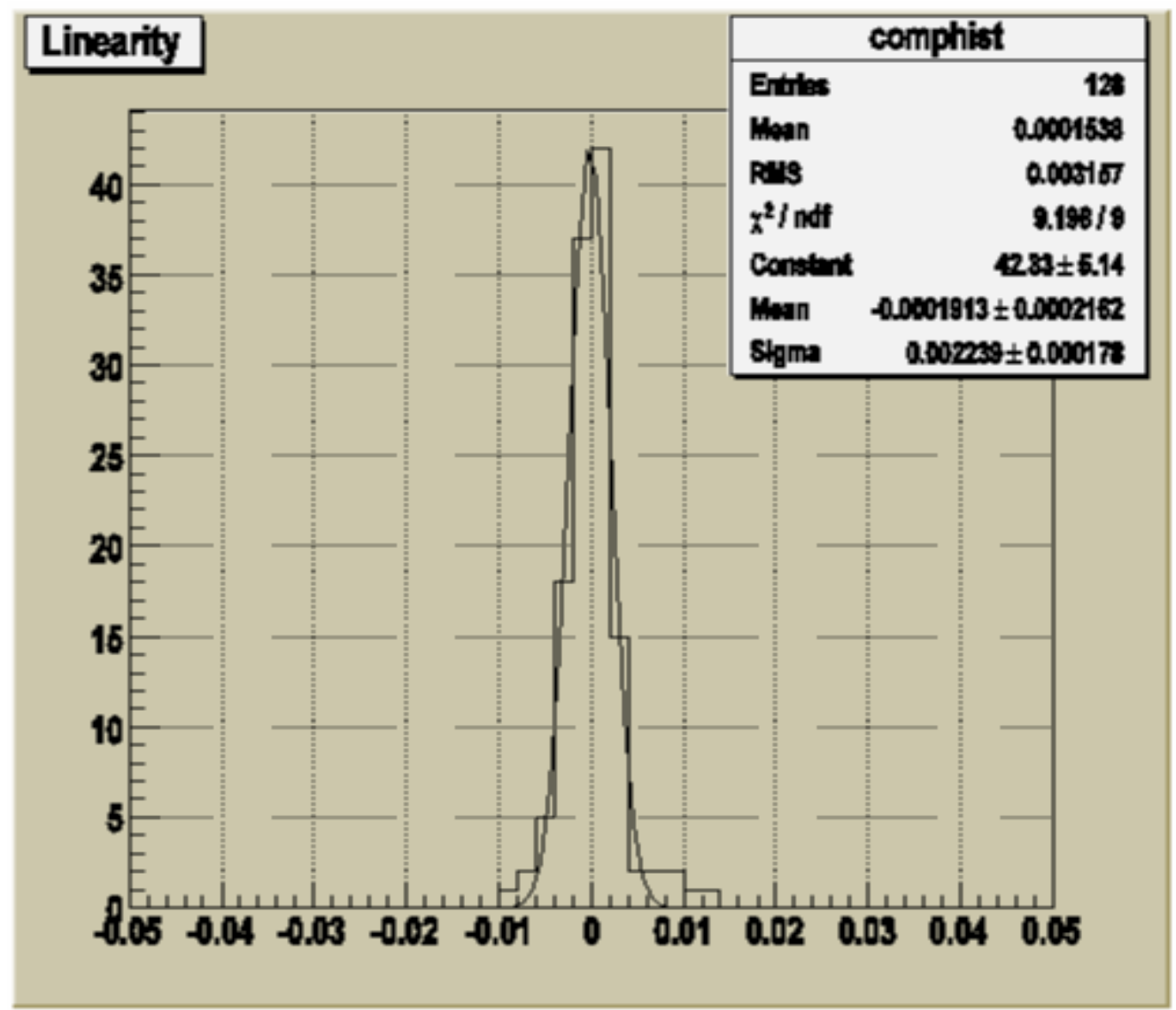

Figure 4: A histogram of (ADC - ADC_fit)/ADC_fit.

\section{Caution for the CAMAC power supplies}

Two power supplies of the CAMAC chasses, one for the old "BIRA" and another one for the "DSP", have failed so far. On the shaper board, one may put in a terminator to switch on/off the source input. With 12 WFD's in a CAMAC chassis, switching on the source input would increase the current reading on the CAMAC chassis about $3 \mathrm{~A}$. The total current in the chassis with 12 WFD's is easily 50 A or above. But 50 A is typically the maximum current that these CAMAC power supplies can support and it is not surprising the power supply mail fail after some time.

Apparently, the newer "BIRA" CAMAC chassis can support up to 60 A according to A. Casper in HEEP. It is also interesting to note that with the newer "BIRA" CAMAC chassis takes less current than the "DSP". With 12 WFD's all on with attenuation set at 5, the "DSP" chassis takes about 49 A whereas the newer "BIRA" takes only about 43 A. Probably also because of this reason, the Xilinx FPGA's of the WFD's on the latter "BIRA" chassis do not get the firmware transferred to them from the 
EPROM on the WFD's every time when the chassis power cycled. They typically need a CAMAC command (0x8 to A8F16) to trigger the firmware transfer.

${ }^{\mathrm{i}}$ http://www.yale.edu/fastcamac/.

ii http://www.wiener-d.com/products/17/28.html.

iii http://www.cmcamac.com/.

${ }^{\text {iv }}$ http://www.lecroy.com/lrs/dsheets/2367.HTM.

${ }^{\mathrm{v}}$ https://www.scientificlinux.org/.

${ }^{\text {vi }}$ http://www.agsrhichome.bnl.gov/Controls/doc/usingCdev/remoteAccessCdevData.html.

${ }^{\text {vii }}$ From the Corporate history at http://www.lemo.com/about/history.jsp, it says: “LEMO®, founded in Switzerland in 1946, took its name from the company founder, engineer Léon Mouttet. Originally a manufacturer of contacts in noble and rare metals, the company took a major step forward in 1957 with the introduction of the push-pull self-latching connector." 